\title{
Theoretical study of crossed and parallel carbon nanotube junctions and three-dimensional grid structures
}

\author{
S. Dag, ${ }^{1}$ R. T. Senger, ${ }^{1,2}$ and S. Ciraci ${ }^{1}$ \\ ${ }^{1}$ Department of Physics, Bilkent University, 06800 Ankara, Turkey \\ ${ }^{2}$ TÜBITAK-UEKAE, P.K. 74, 41470 Gebze, Kocaeli, Turkey
}

(Received 19 May 2004; published 10 November 2004)

\begin{abstract}
This work presents a first-principles study of parallel and crossed junctions of single-wall carbon nanotubes (SWNT). The crossed junctions are modeled by two-dimensional grids of zigzag SWNTs. The atomic and electronic structure, stability, and energetics of the junctions are studied for different magnitudes of contact forces pressing the tubes towards each other and hence inducing radial deformations. Under relatively weak contact forces the tubes are linked with intertube bonds which allow a significant conductance through the junction. These interlinking bonds survive even after the contact forces are released and whole structure is fully relaxed. Upon increasing contact force and radial deformation the tube surfaces are flattened but the interlinking bonds are broken to lead to a relatively wider intertube spacing. The intertube conductance through such a junction diminish because of finite potential barrier intervening between the tubes. The linkage of crossing tubes to form stable junctions is enhanced by a vacancy created at the contact. The three-dimensional grid structure formed by SWNTs is also investigated as a possible framework in device integration.
\end{abstract}

DOI: 10.1103/PhysRevB.70.205407 PACS number(s): 73.22.-f, 73.63.Fg, 73.63.Rt, 61.46.+w

\section{INTRODUCTION}

The intensive research on carbon nanotubes ${ }^{1}$ has revealed a variety of properties ${ }^{2-11}$ which make them important in nanoscience and nanotechnology. In particular, carbon nanotubes can be semiconducting or metallic depending on their chirality and radius. ${ }^{2-6}$ Moreover, their electrical and magnetic properties can be modified by external agents; namely by functionalization ${ }^{11}$ and by radial strain. ${ }^{11-14}$ Their reactivity to foreign molecules and atoms is enhanced to a large extent by their curvature. ${ }^{4,15}$ Several atoms are shown to be adsorbed on the tube surface with significant binding energies. ${ }^{16}$ While they are very strong axially and have a high Young's modulus, they are very flexible radially and can sustain high radial deformation. ${ }^{17,18}$ It has been shown that a semiconducting tube becomes metallic as a result of radial deformation transforming the circular cross section into an elliptical one. ${ }^{14,18}$

Thus single-wall carbon nanotubes (SWNT) have been considered as a major nanostructure for future nanoscale electronics. ${ }^{10,11,19-21}$ Not only various devices to be fabricated from SWNTs, but also SWNT based interconnects have been of interest. ${ }^{22,23}$ Recent theoretical analyses have shown that electronic devices together with their metallic interconnects can, in principle, be fabricated on a single tube. ${ }^{14}$

Parallel, cross, and Y junctions of carbon nanotubes, because of their unusual physical properties, have been studied experimentally and theoretically. ${ }^{24-39}$ Based on generalized tight-binding molecular dynamics (MD) calculations Menon and Srivastava ${ }^{24}$ proposed that stable $\mathrm{T}$ junction of SWNTs can form the smallest prototypes of microscopic metalsemiconductor-metal contact. Yildirim et al. ${ }^{26}$ investigated the character of link between tubes in SWNT ropes under pressure. In addition to the van der Waals packing they found two more different phases with local minima where the link- age is provided by $\mathrm{C}-\mathrm{C}$ bonds between adjacent parallel zigzag SWNTs. However, similar interlinking $\mathrm{C}-\mathrm{C}$ bonds did not form between the $(6,6)$ parallel tubes even if they are deformed under a very high pressure. Terrones et al. ${ }^{34}$ have fabricated stable junctions of various geometries $(+, \mathrm{X}, \mathrm{T}, \mathrm{Y})$ in-situ in a transmission electron microscope. Electron beam exposure at high temperatures induced structural defects which promoted the joining of tubes. Classical MD calculations have been carried out to simulate various junctions of SWNT. ${ }^{33,34}$ Employing empirical potential MD, Krasheninnikov et al. ${ }^{35}$ simulated the bombardment of nanotubes and demonstrated that crossed nanotubes can be welded. Recently, Yoon et al. ${ }^{30}$ presented a first-principles study of deformation and quantum electronic conductance of junctions formed by two crossed $(5,5)$ metallic SWNTs. Despite, high contact forces, the $\mathrm{C}-\mathrm{C}$ bonds between these tubes did not form to link the junction.

The conductance through nanotube junctions has been also a subject of interest. Using an atomic force microscope, Postma et al. ${ }^{29}$ manipulated SWNTs to create a junction such as buckles and crossings within individual metallic SWNT connected to metallic electrodes. They showed that these manipulated structures behave as tunnel junctions. By changing the angular alignment of the atomic lattices at the SWNTgraphite contact it has been shown that the contact resistance can be varried by more than an order of magnitude in a controllable and reproducible manner, indicating that momentum conservation also dictates the junction resistance. ${ }^{28}$ Buldum and $\mathrm{Lu}^{32}$ carried out electron transport calculations through the junction of two crossing SWNTs. By rotating one of the tubes they found that intertube conductance is strongly dependent on the atomic registry between two tubes. It is now well understood that the junctions of SWNTs exhibit novel electronic properties so that they can be ideal nanostructures to fabricate robust molecular scale electronics. ${ }^{27,31}$ As a kind of nanotube contact, the geometry 
of telescopic arrangement of two shells of a MWNT have been recently considered for calculating inter-shell currents. ${ }^{40}$ They find a trend of a quasi-linear increase in the inter-shell conductance with the increase in the length of the overlap region.

All previous studies have indicated the importance of nanotube junctions in device applications and brought about issues to be addressed both theoretically and experimentally. In particular, the description of the atomic structure of the contact between two tubes as a function of contact forces (or uniaxial stress), and electronic energy structure and electronic potential have needed a detailed ab-initio analysis. It is now important to know under what circumstances crossednanotubes remain attached (or welded) to each other and the junction becomes a conductor. Also questions as to the roles of the atomic registry forces pressing the tubes to induce radial deformation and point defects at the contact in forming junctions have remained to be clarified.

In this paper we present a detailed, first-principles analysis of the junction of crossed and parallel semiconducting SWNTs in different atomic registries as a function of uniaxial stress pressing the tubes. We examined their energetics, stability and electronic properties. As a first-step in three-dimensional (3D) device integration based on SWNTs, we also examined a 3D grid made by the periodical stacking of SWNTs. Our results are summarized as follows: (i) The intertube interactions and resulting electronic properties are strongly dependent on the radial deformation of the tubes at the contact. They do not vary continuously, but exhibit various phases depending upon the strength of the contact forces. (ii) A vacancy created at the contact promotes the linking through $s p^{3}$-like bonds forming between tubes. (iii) These $s p^{3}$-like bonds link crossing tubes not only mechanically, but also electronically, and may survive even after the contact forces are released.

The organization of this paper is as follows. In Sec. II, we first present the computational details which are used in our study. In Sec. III, we specify various junctions and give a detailed analysis of their atomic and electronic structures. The 3D grid structure is discussed in Sec. IV. In Sec. V, the results of our transport calculations are presented.

\section{COMPUTATIONAL MODEL AND METHOD}

First-principles total energy and electronic structure calculations have been performed using the pseudopotential plane wave method ${ }^{41}$ within the generalized gradient approximation (GGA). ${ }^{42}$ We treated both crossing and parallel junctions of $(8,0)$ zigzag tubes within the supercell geometry as described in Fig. 1. Two crossed tubes (also a crossbar structure) are modeled by parallel rows of $(8,0)$ tubes along the $x$ direction which are placed on similar but perpendicular rows of $(8,0)$ tubes along the $y$ direction. This way, a $2 \mathrm{D}$ square grid of crossed tubes is generated. These grids are repeated periodically along the $z$-direction with a vacuum spacing of $10 \AA$ between grids. Owing to this large vacuum spacing and supercell lattice parameters $a=b=12.76 \AA=[3$ $\times$ lattice parameter of $(8,0)$ tube, $\left.c_{S W N T}\right]$ along the $x$ and $y$ directions the coupling of adjacent junctions of the grids is
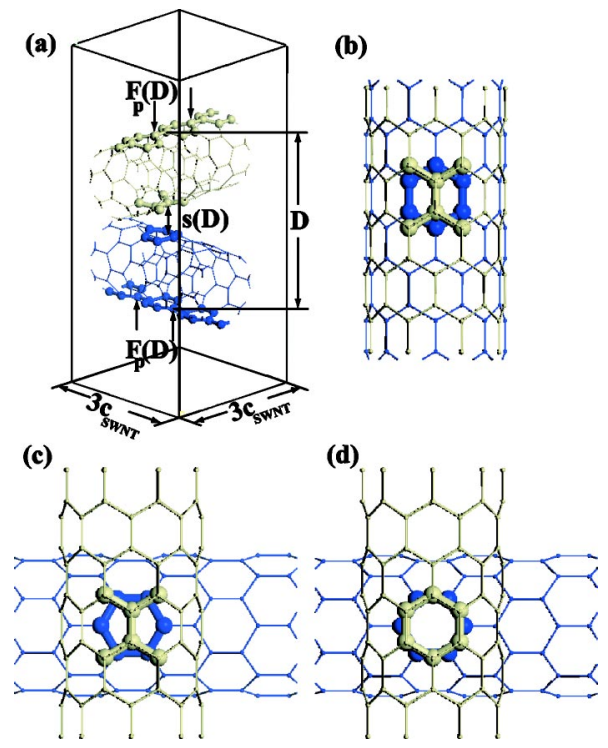

FIG. 1. (a) Supercell used to simulate a junction of two crossed tubes. $F_{p}(D)$ is the contact force generated due to a fixed distance $D<D_{v d W}$, and $s(D)$ is optimized spacing between the surfaces of two SWNTs at the contact. (b) Bridge-Hollow (B-H) atomic registry between two parallel zigzag SWNT, where the $\mathrm{C}-\mathrm{C}$ bonds of top SWNT along its axis face the hexagon of the bottom SWNT. (c) The same as (b) for the crossbar structure. (d) Hexagon-hexagon $(\mathrm{H}-\mathrm{H})$ atomic registry for the crossbar structure. The lattice parameter of the bare $(8,0)$ tube is $c_{S W N T}=4.25 \AA$.

expected to be small. However, the tube sides between two adjacent junctions can be affected from the radial deformation of the contact. In a crossbar having free ends, the circular cross section of the bare tube is expected to be recovered after some distance from the junction. As for the junction of parallel tubes, they are modeled by two infinite parallel $(8,0)$ SWNTs in contact.

Normally, two crossed or parallel, stress free tubes are linked with a very weak van der Waals interaction with a spacing $s_{v d W} \sim 3.3 \AA$. Then, the distance from the top of one tube to the bottom of the other one, $D=D_{v d W} \cong s_{v d W}+2 R_{1}$ $+2 R_{2}$, where $R_{1}$ and $R_{2}$ are radii of free tubes. In order to create contact beyond van der Waals linkage, carbon atoms located at the top and bottom of the junction are fixed at a given distance, $D<D_{v d W}$ as indicated in Fig. 1. This situation is equivalent to generate contact forces $F_{p}(D)$ which press two tubes towards each other. The rest of the atoms in the supercell are relaxed to minimize the total energy. At the end, the tubes in contact are deformed radially to have elliptical cross sections and a spacing $s(D)$ between the tubes is achieved after the relaxation. By changing $D$ different contact forces $F_{p}(D)$ yielding different spacings $s(D)$ are obtained to examine the effect of the deformation at the junction. In the force-free calculations, all the atoms in the supercell have been relaxed.

We considered two different atomic registries at the contact as described in Fig. 1: (i) the H-H registry where a hexagon on one tube lies over a hexagon on the other tube; (ii) the $\mathrm{B}-\mathrm{H}$ configuration where one $\mathrm{C}-\mathrm{C}$ bridge-bond along the axis of the tube faces a hexagon on the other tube at the center of contact. By removing the vacuum spacings 


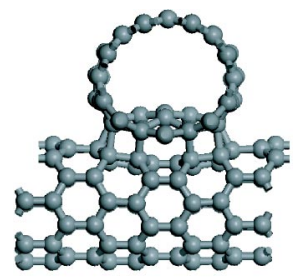

H-H

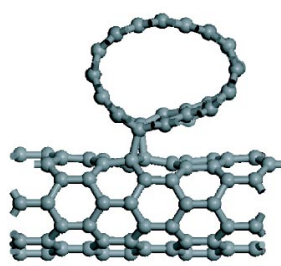

B-H2
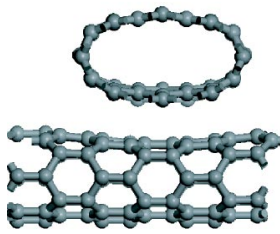

B-H4
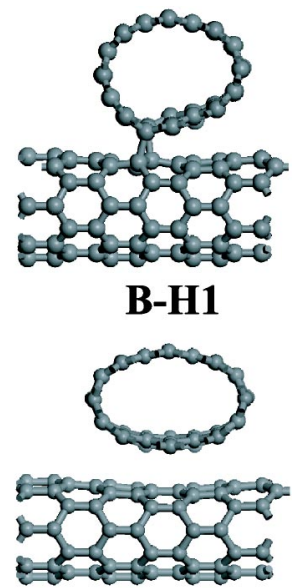

B-H3

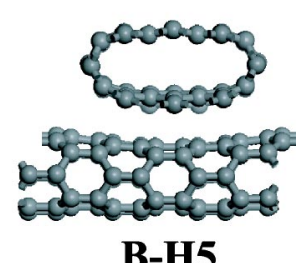

B-H5

FIG. 2. Relaxed atomic structures of two crossed $(8,0)$ SWNTs with different atomic registries $(\mathrm{H}-\mathrm{H}$ and $\mathrm{B}-\mathrm{H})$. B-H junction has been studied for five different spacing values of $s(D)$ labeled by B-H1, B-H2, B-H3, B-H4 and B-H5.

between adjacent 2D grids, 3D grid structures (where each tube is now in contact with two perpendicular tubes from above and below) have been constructed. We used 5 k-points within the Monkhorst-Pack ${ }^{43}$ special k-point scheme in the sampling of the Brillouin zone of the 2D grid. For the 3D grid structure 14 special $\mathbf{k}$-points have been used.

Since the main objective of this study is to reveal the electronic properties of SWNT junctions, we carried out an extensive analysis for the quantum transport of electrons. To this end, we used nonequilibrium Green's function formalism together with an empirical tight-binding method to investigate the electron transport from one finite tube to the other one through the junction. Each finite tube is attached to a different reservoir. The details of the method are explained in Sec. V.

\section{ATOMIC AND ELECTRONIC STRUCTURE OF JUNCTIONS}

\section{A. Junctions of crossed SWNTs}

We considered 2D grids having either $\mathrm{H}-\mathrm{H}$ or $\mathrm{B}-\mathrm{H}$ atomic registry at the contact region. For the $\mathrm{H}-\mathrm{H}$ registry, we studied only one junction which has $D=12.53 \AA$. The relaxed junction together with $\mathrm{C}-\mathrm{C}$ bonds connecting two SWNTs is illustrated in Fig. 2. The circular cross sections of tubes are significantly deformed. The deformation energy is calculated as the difference between the total energy of two noninteracting bare tubes and the total energy of crossed ones and is found to be $19.1 \mathrm{eV}$ per supercell (or per 6 unit cells of SWNT). The electronic band structure of the corresponding

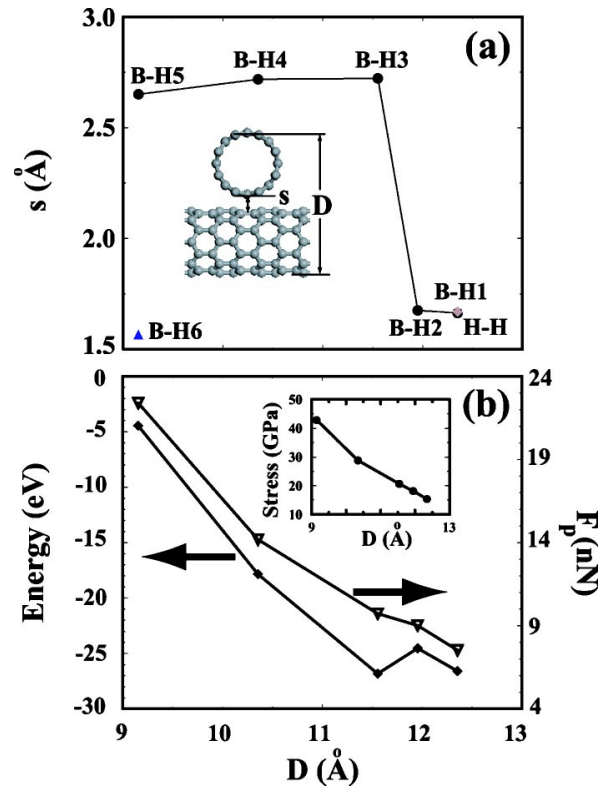

FIG. 3. (a) Variation of relaxed spacing $s$, between two crossed nanotubes and (b) its energy (shown by diamonds) and contact force $F_{p}$ (shown by triangles) as a function of $D$. The stress per supercell and atomic configuration of the junction are shown by insets. In (a) filled circles, light diamonds and triangles indicate $\mathrm{B}-\mathrm{H}, \mathrm{H}-\mathrm{H}$ registries and the $\mathrm{B}-\mathrm{H}$ registry including a single vacancy, respectively. In (b) diamonds and triangles are joined by lines as a guide to the eye; but the detailed structure of possible local minima are omitted.

2D grid structure in the $\mathrm{H}-\mathrm{H}$ registry is semiconducting and has a band gap of $\sim 0.3 \mathrm{eV}$.

Junctions of crossed $(8,0)$ tubes in the B-H registry have been treated for five different values of $D$ as illustrated in Fig. 2. As one goes from junction B-H1 to B-H5, the distance $D$ has decreased gradually $(12.35,11.95,11.55,10.35$ and $9.15 \AA$, respectively). While $D$ is reduced from $12.35 \AA$ to $9.65 \AA$, some physical properties have displayed irregular changes. Figure 3 shows variations of the spacing, energy and contact forces with $D$; namely $s(D), E_{T}(D)$ and $F_{p}(D)$, respectively. It should be noted that $F_{p}(D)=0$, for values of $D$ that make $E_{D}$ a local minimum. The changes of the spacing, bonding and atomic structure, as well as the electronic structure are nontrivial, even paradoxical. For example, B-H1 and B-H2 junctions have small spacings $[s(D) \sim 1.7 \AA]$, which allow the bond formation between the crossed tubes. The tubes by themselves display high curvature, where the $\mathrm{C}-\mathrm{C}$ bonds can form between their surfaces. On the other hand, in spite of smaller $D$ and stronger $F_{p}$ in the B-H3-B-H5 junctions, their spacings $s(D)$ increase to $\sim 2.7 \AA$ and the interlinking bonds at the contact are broken. This situation arises due to the flattening of the curved tube surfaces at the junction. While contact atoms in B-H1 and $\mathrm{B}-\mathrm{H} 2$ geometries are forming $s p^{3}$-like bonds between the tube surfaces, the flattened surfaces of B-H3-B-H5 junctions behave more graphite-like with large intertube spacings and finite potential barrier $\Phi_{B}(z=s / 2)=V_{e}(z=s / 2)-E_{F}>0$ (where $V_{e}$ is electronic potential energy).

Depending on the value of $s(D)$ and the presence of interlinking bonds, we distinguish two different types of junc- 


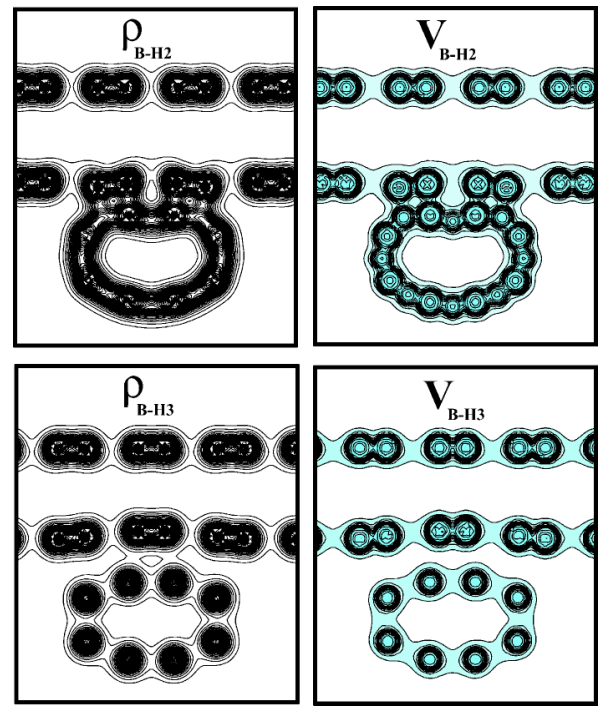

FIG. 4. Contour plots of total charge density $\rho_{T}$ and SCF electronic potential $V_{e}$ of $\mathrm{B}-\mathrm{H} 2$ and $\mathrm{B}-\mathrm{H} 3$ junctions. In the right panels the potential energy in the white regions is higher than the Fermi energy, i.e. $\Phi_{B}>0$.

tions. Junctions such as B-H1 and B-H2 have established electronic contact between the tubes, while in B-H3-B-H5 junctions $s(D)$ increases and interlinking bonds at the contact are broken. These cases can be analyzed by charge density, $\rho_{T}(\mathbf{r})$, and electronic potential energy, $V_{e}(\mathbf{r})$, contour plots as shown in Fig. 4. For example, small spacing $s(D) \sim 1.6 \AA$ of $\mathrm{B}-\mathrm{H} 2$ junction allows the formation of $\mathrm{C}-\mathrm{C}$ bonds between the surfaces of two SWNTs. These interlinking bonds are easily distinguished in Fig. 4, whereas in the B-H3 junction $s(D)$ has increased to $\sim 2.7 \AA$, and a finite potential barrier $\Phi_{B}$ has developed between two tubes.

Important features of the electronic structure of the junctions are revealed from the electronic band structure of the corresponding 2D grid structure along the $k_{x^{-}}$and $k_{y}$-direction. The grid formed of $\mathrm{H}-\mathrm{H}$ junctions is a semiconductor with a band gap of $E_{g}=0.3 \mathrm{eV}$ in spite of the C-C bonds which connect crossing SWNTs. Similarly, the 2D grids of $\mathrm{B}-\mathrm{H} 1$ and $\mathrm{B}-\mathrm{H} 2$ junctions are semiconductor, but the band gap gets gradually smaller. It is $0.17 \mathrm{eV}$ in the former, but is reduced to $0.1 \mathrm{eV}$ in the latter. The gap is closed in $\mathrm{B}-\mathrm{H} 3$ and B-H4 structures owing to a relatively stronger deformation of tubes at the contact. Interestingly, upon further decreasing of $D$, the gap opened again and hence 2D grid becomes again a semiconductor. The metallicity at the intermediate levels of deformation is due to the radial deformation of each tube at the contact. Because of $\sigma^{*}-\pi^{*}$ hybridization, $4,14,18$ the conduction band of the $\pi^{*}$-singlet states dips below the Fermi level of both SWNT. First closing then opening of the band gap of the $2 \mathrm{D}$ grid structure is a behavior specific to the junction of crossed $(8,0)$ tubes. Note that the metallization of the grid is due to a relatively short distance (approximately one unit cell of SWNT) between two adjacent nodes. If the edges of the squares of the grid were taken long enough, the central regions of SWNT between two nodes would be unaffected and remain undeformed in spite of the severe radial deformation at the con-
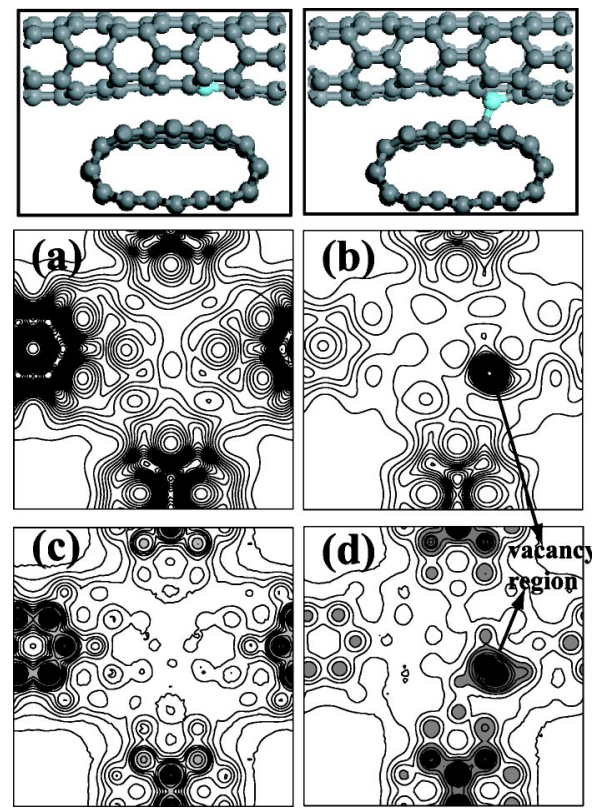

FIG. 5. Relaxed atomic structure, total charge density $\rho_{T}$ and SCF-electronic potential $V_{e}$ of the junction of crossed SWNTs. Left panels: B-H5 contact; right panels: B-H5-type contact including a single carbon vacancy. (a) and (b) are charge density contour plots on a lateral plane bisecting the spacing $s$ between tubes. (c) and (d) are the same for the electronic potential energy. While $V_{e}>E_{F}$ at the contact and hence $\Phi_{B}>0$ in (c), the potential barrier is collapsed and an orifice is formed between two tubes through the contact in (d).

tacts. Under these circumstances the 2D grid would maintain its gap. On the other hand, the edges of the 2D grid made by metallic SWNTs (such as armchair tubes) is expected to remain metallic no matter what the character of the junction is. The conductivity is then controlled by the contact resistance of the metallic tubes.

\section{B. Effect of vacancy and carbon impurity}

Crossbars have been produced by the exposure of the junction to the electron beam, where one generates several imperfections. ${ }^{33,34}$ As a possible imperfection we considered the effect of a vacancy existing on one of the tubes at the contact region. In spite of a high deformation and small $D$ in the B-H5 junction the spacing between the tubes has been rather large. After creating a vacancy on the surface of one of the tubes the bonding character near the vacancy has been changed from a $s p^{2}$ - to a $s p^{3}$-like configuration; thereafter the spacing has decreased and eventually an interlinking bond has formed. It appears that an imperfection like a vacancy at the contact provides an electronic charge distribution and atomic structure which are suitable for linking of two tubes. The linkage and eventually welding (or merging) of two tubes at the junction can take place by the creation of a large number of vacancies or divacancies. Interestingly, we found that the 2D grid having a single vacancy at the contact is semiconducting with a band gap $E_{g} \sim 0.25 \mathrm{eV}$.

The effect of vacancy is further examined in Fig. 5 by comparing total charge density and SCF-potential in a plane 
bisecting $s(D)$ between the tubes at the junction. In the absence of vacancy B-H5 junction has low $\rho_{T}$, but $V_{e}>E_{F}$ in the same plane. A finite barrier $\Phi_{B}$ develops, and prevents electrons from the ballistic motion between the tubes. The situation is, however, different if a vacancy is incorporated to one of the tubes at the contact. In the region of the interlinking $\mathrm{C}-\mathrm{C}$ bond induced by the vacancy, we see high charge density and low potential. This situation is reminiscent of the fact that the interlinking $\mathrm{C}-\mathrm{C}$ bond made an orifice or hole through the potential barrier. Such an orifice can allow the ballistic electron transfer if its diameter is large so that an effective barrier does not develop due to size effects.

A single carbon atom placed between two tubes and on top of the mutual axial bonds crossing at the contact can form four directional and strong bonds. The interlinking of the tubes is found to be favorable energetically. We found the total energy of the whole system is lowered by $4.6 \mathrm{eV}$. This means that an energy of $4.6 \mathrm{eV}$ is required to disconnect the crossed $(8,0)$ tubes linked by a single carbon atom.

\section{Parallel tubes}

The junctions made by parallel tubes may display a behavior which is slightly different from the crossing SWNTs. Earlier Yildirim et al. ${ }^{26}$ examined the interlinking of SWNTs forming a $2 \mathrm{D}$ hexagonal lattice under pressure. In addition to the interlinking of tubes via van der Waals attractive interactions under zero pressure, they found two different local minima of the Born-Oppenheimer surface at different ranges of applied pressure. In the phases corresponding to these minima, $\mathrm{C}-\mathrm{C}$ bonds have formed to link the tubes in one direction in the first minimum and in two directions in the second minimum. The present structure and model differs from those of Yildirim et al. ${ }^{26}$ since only two parallel tubes are considered in contact. Two free SWNTs are expected to be linked by the van der Waals interaction with $s_{v d W}$ $\sim 3.3 \AA$ and $D_{v d W} \cong s_{v d W}+2 R$. By constraining them with $D<D_{v d W}$, we see that interlinking bonds are easily formed between two tubes for low $F_{p}$. In contrast to what one sees in the junctions of crossing tubes, these bonds continue to exist even under strong contact forces. The spacing $s(D)$ is in the range of $\sim 1.6 \AA$ no matter what the value of $D$ is. Each interlinking bond pulls and connects two $\mathrm{C}$ atoms, one from each tube, and changes the local $s p^{2}$-type bonding to $s p^{3}$-like bonding configuration (see Fig. 6).

\section{Free junctions}

Having examined the energetics and atomic structure of junctions of crossing and parallel tubes, we next address the question as to what happens if the contact force is released and hence the tubes are left free. It is important to know whether the junction survives or the linking of tubes ceases. To this end, we optimized B-H2 and B-H3 junctions after $F_{p}(D)$ is released (or the constraint due to $D$ is lifted). Note that under contact forces, the former had interlinking bonds, but the latter had relatively larger $s(D)$ whereby interlinking bonds were broken. Once the $F_{p}$ is released from the B-H2 junction, the deformation of SWNTs steadily relaxed to re-
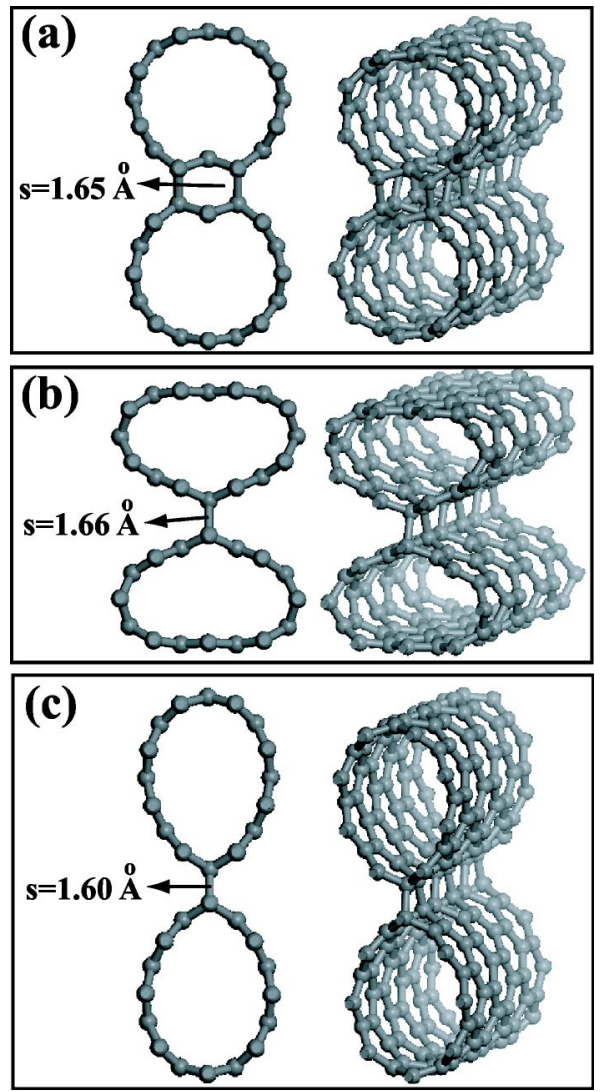

FIG. 6. Relaxed atomic structure of the junctions between two parallel tubes under different contact force or $D$. (a) Large $D$ and hence weak deformation. (b) Small $D$, hence strong deformation. (c) Relaxed junction after the contact force in (b) is released. Parallel tubes have $\mathrm{B}-\mathrm{H}$ registry.

assume their original bare circular shape. After the full relaxation the $\mathrm{C}-\mathrm{C}$ bonds continued to link two SWNTs, but the form of the cross section at the contact region has changed and the upper tube has rotated as shown in Fig. 7. The curvature increased locally at both ends of interlinking $\mathrm{C}-\mathrm{C}$ bonds to comply with the $s p^{3}$-like bond configuration. It appears that the B-H2-type junction forming under weak $F_{p}$ can provide a connection between crossed SWNTs as a local minimum. However, the B-H3 junction behaves differently upon lifting of the contact forces. Without being captured in any local minimum, cross sectional deformation is gradually eliminated and eventually two SWNTs become disconnected.

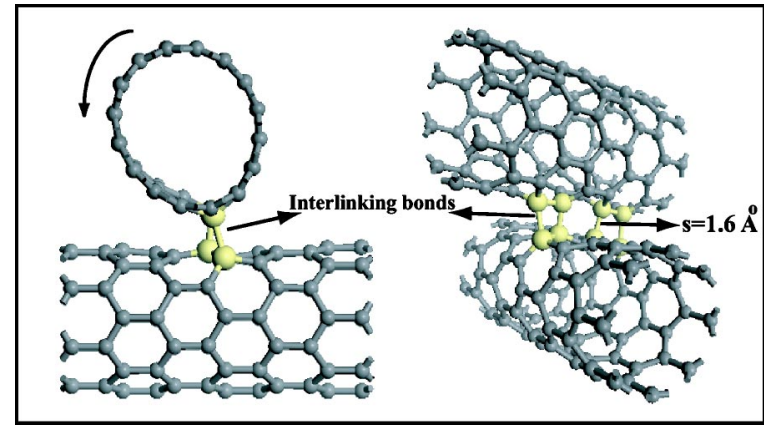

FIG. 7. Relaxed atomic structure of a junction B-H2 after the contact forces $F_{p}$ are released. 
In the case of parallel junctions two tubes remained connected with interlinking $\mathrm{C}-\mathrm{C}$ bonds as shown in Fig. 6(c) even after the contact force is released.

In closing this section we note that Yoon et al. ${ }^{30}$ investigated the junction of crossed $(5,5)$ SWNTs by performing a constrained total energy minimization within a supercell structure, in which only the positions of the atoms near the junction are relaxed while fixing the center-to-center intertube distance at the boundaries to produce the desired contact force. By using the Landauer-Büttiker ${ }^{45,46}$ formula they calculated intertube and intratube conductance as a function of the contact force. These calculations ${ }^{30}$ of crossed nanotube junctions differ from the present one in many respects. First of all the $(5,5)$ metallic tubes considered by Yoon et al. ${ }^{30}$ do not form the interlinking $\mathrm{C}-\mathrm{C}$ bonds between the tubes. Consequently, the large spacing $(s \cong 3.35 \AA)$ occurring in the absence of the contact force, is reduced only to $\sim 2.5 \AA$ under strong contact force. Although this spacing is still too large to form $\mathrm{C}-\mathrm{C}$ interlinking bonds, it may allow a significant wave function overlap of individual tubes at the Fermi level and hence may enhance the tunnelling current as in fact found by Yoon et al. ${ }^{30}$ In the present study $(8,0)$ zigzag tubes can form $\mathrm{C}-\mathrm{C}$ bonds at the contact which, in turn, may lower or even collapse the tunnelling barrier. For the same reason the variation of $s$ with $F_{p}$ is more complex and varies from $3.35 \AA$ (van der Waals linking not included in this study) to $\sim 1.6 \AA$. Our work also differs from the earlier one by the constrains which create the contact force between crossed tubes. Similar to the experimental condition using an Atomic Force Microscope, we pressed the tubes from top and bottom (namely by fixing only a few atoms designated in Fig. 1 at a desired distance) and relaxed the rest of the atoms. As a result, the deformation of the tubes are more realistic, in particular, for the case of strong $F_{p}$.

\section{3D GRID STRUCTURE}

The 3D grids of SWNTs add an additional dimension to the planar structures and may be of use in 3D integration and other similar applications such as forming a $3 \mathrm{D}$ periodic framework for the artificial crystal structure of giant molecules. Here we studied a 3D grid made of a supercell containing 6 unit cells of the $(8,0)$ SWNT. As described in Sec. II, our model for the 3D grid structure shown in Fig. 8 is generated by stacking the B-H1 junctions along the $z$-axis. The contact force is imposed by fixing the supercell parameter along the $z$-axis at a specific value, which leads to certain deformation of tubes upon relaxation. Owing to the initial structure and the supercell parameters, the SWNTs along the $y$-axis form interlinking bonds with the SWNTs along the $x$-axis from only one side. At the other (opposite) side of SWNTs the spacing $s$ between adjacent tubes is large and does not allow any interlinking bonds. Accordingly, a potential barrier develops which prevents the ballistic electron transfer along the $z$-axis. Such a one-sided linking of tubes at the junction appears to be circumstantial, however. Under different $F_{p}$ either two-sided linkings or two-sided detached junctions with large $s$ may occur.

The electronic band structure shown in Fig. 8 confirms the situation that the $3 \mathrm{D}$ grid structure is electronically discon-

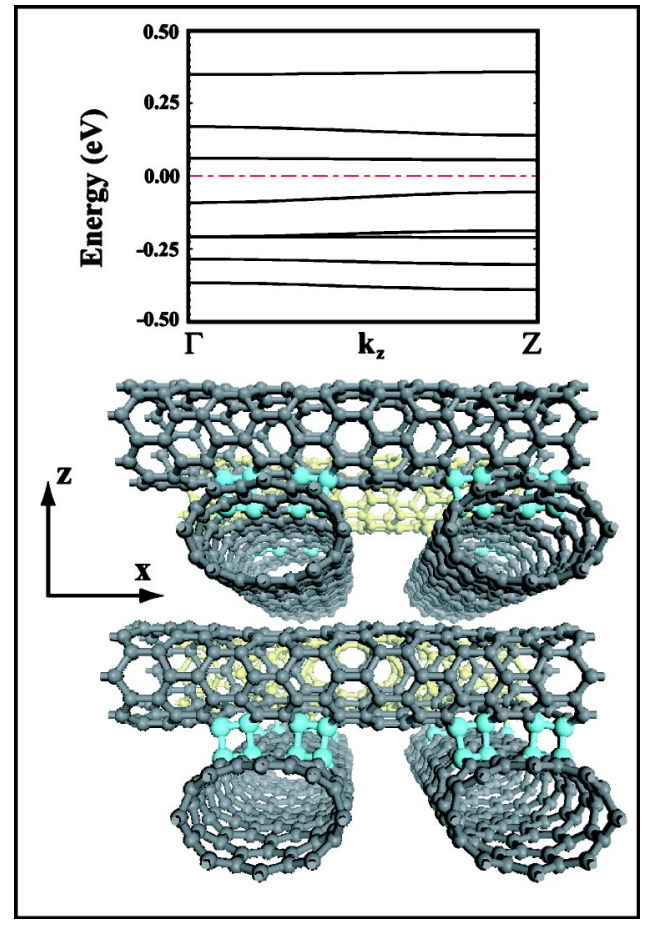

FIG. 8. Energy band structure along the $z$-axis shown by an inset, and the relaxed atomic structure of the corresponding $3 \mathrm{D}$ grid of the $(8,0)$ zigzag tubes.

nected along the $z$ axis. It has a band gap of $0.15 \mathrm{eV}$, and flat bands. We, however, note that the electronic and mechanical properties of the grid structure can be tuned by changing the supercell parameters. The electronic and mechanical linking of SWNTs along $x, y$, and $z$ directions depend on the contact forces inducing radial deformation, and on the lateral lattice parameters. Upon releasing the contact force, the interlinking bonds can provide stability and may lead to metallic properties. Nevertheless, the model discussed here demonstrates that the stable 3D grid and crystal structure can, in principle, be formed from SWNTs. These 3D grid structures can be modified by external agents, such as stress, modulating absorption of molecules and atoms. For example, through the decoration of transition metal atoms or magnetic molecules the grid can gain magnetic properties. 3D grids made by armchair tubes have metallic interconnects between nodes.

\section{ELECTRON TRANSPORT}

The rapid advances in the measurements of electrical conductance of individual molecular- and atomic-sized devices require commensurate advances in the theoretical understanding of the detailed microscopic mechanisms. Modeling of a single element of nanodevices is needed to provide interpretations to predict device characteristics. Several approaches have been developed to calculate the quantum conductance in nanostructures, based on semiempirical (tightbinding, Hückel) models. More recently, a variety of firstprinciples formulations have appeared. Ab initio approaches have also been extensively used to characterize the electrical transport properties of nanostructures. 


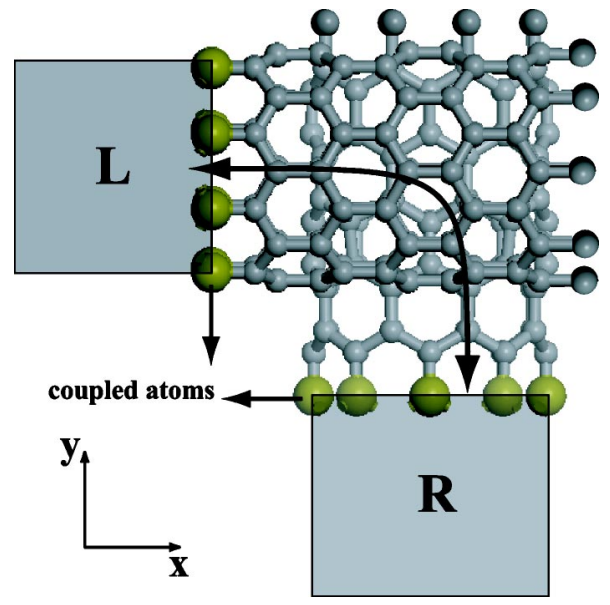

FIG. 9. An atomistic model which describes the electronic transport through the junction. $\mathbf{L}$ and $\mathbf{R}$ are reservoirs where finite tubes forming the junction are coupled to.

In the present study to analyze the conductance properties of junctions of two SWNTs we used the Green's function technique combined with the Landauer-Büttiker formalism and a parametrized tight binding model. ${ }^{44}$ The junction is taken to be coupled to two semi-infinite electrodes (reservoirs) from the end surfaces $L$ and $R$ as depicted in Fig. 9.

The conductance through the junction is given by the Landauer type formula $\mathrm{a}^{45,46}$ in terms of the Green's functions of the junction and the coupling of the junction to the electrodes; ${ }^{47}$

$$
G=\frac{2 e^{2}}{h} \operatorname{Tr}\left(\Gamma_{L} G^{r} \Gamma_{R} G^{a}\right),
$$

where $G^{r}$ and $G^{a}$ are the retarded and advanced Green's functions of the conductor and $\Gamma_{L}$ and $\Gamma_{R}$ are the coupling functions of the conductor to the electrodes. The retarded Green's function is given by the expression

$$
G^{r}=\left(\epsilon-H-\Sigma_{L}-\Sigma_{R}\right)^{-1},
$$

where $H$ is the Hamiltonian of the conductor region, and $\Sigma_{L}$, $\Sigma_{R}$ are the self-energy terms due to the electrodes. The selfenergies and the coupling functions are related through $\Gamma_{L(R)}=-2 \operatorname{Im} \Sigma_{L(R)}$. In this approach the properties and the effects of the electrodes are represented by the self-energy terms which we have parametrized by the corresponding line-width functions $\left(\Gamma_{L(R)}\right)_{i j}=\gamma \delta_{i j}$, where the indices run through the orbitals of the surface atoms at the contacts. Such parametrization of self-energy terms corresponds to the approximation of the wide-band $\operatorname{limit}^{48}$ where the level shifts, $\operatorname{Re} \Sigma_{L(R)}$, are neglected and the linewidths are taken as an energy-independent constant, $\gamma$, for every level of the surface atoms. After a few trials, in our calculations we chose $\gamma=0.5$ fixed, which provides a sensible broadening of conducton channels shown in Fig. 10.

Within the tight-binding model all these functions are $4 n \times 4 n$ matrices ( $n$ is the number of atoms in the junction region) expressed in terms of the $s, p_{x}, p_{y}, p_{z}$ parametrization of carbon as given in Ref. 44. Here, the selection of range for suitable nearest neighbor interactions in the matrix elements

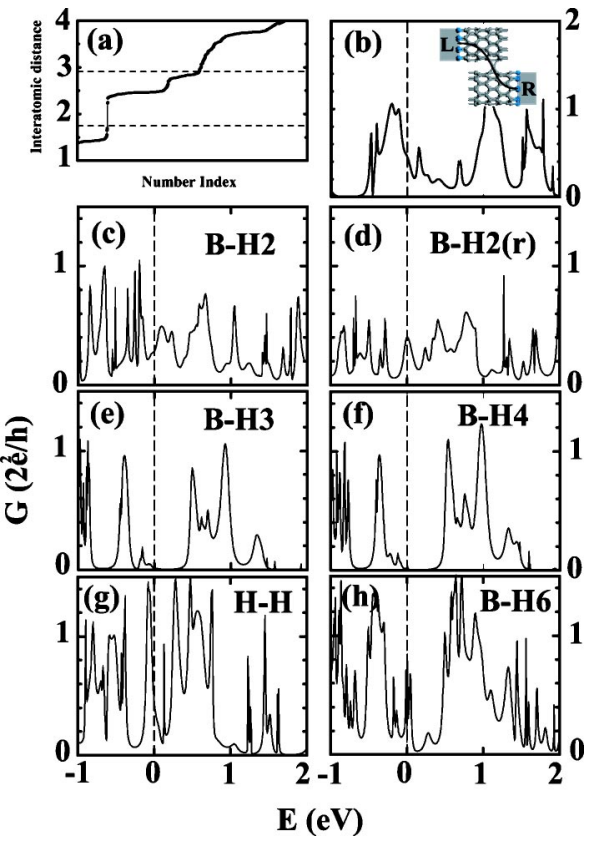

FIG. 10. Calculated conductance $G$ versus energy $E$ for various junctions. (a) Distribution ordering of interatomic distances in the B-H2 junction, i.e., $R_{i j}$ versus the number index of sorted distances. The dashed line at $1.75 \AA$ and $2.91 \AA$ correspond to the domains of tight-binding parametrization; (b) the B-B junction of two parallel tubes connected by a carbon atom; (c) the B-H2 junction of crossed tubes. (d) The B-H2 junction relaxed after contact forces are released; (e) B-H3 junction; (f) B-H4 junction; (g) A junction having $\mathrm{H}-\mathrm{H}$ registry; (h) the B-H6 junction which is B-H5 including a single vacancy. In all plots the coupling parameter is fixed at $\gamma$ $=0.5$. Zero of energy is set at the Fermi level.

$\left\langle\varphi_{i, n}(\mathbf{r})|H| \varphi_{j, m}\left(\mathbf{r}-\mathbf{R}_{i j}\right)\right\rangle$ (or setting a cutoff distance so that $R_{i j}<R_{\text {cutoff }}$ yields a nonzero interatomic interaction) and the appropriate scaling factor of energy parameters are essential. To this end, we examined the distribution of interatomic distances in these structures. Figure 10(a) shows the ordering of interatomic distance $R_{i j}$ in the B-H2 junction. Here we can clearly distinguish three nearest neighbor distances; namely the first nearest neighbor distance with $R_{i j}<1.75 \AA$, and second and third nearest neighbor distances with $R_{i j}<2.91 \AA$. Then, Slater-Koster Hamiltonian parameters of the tightbinding model are as follows: The on-site energies are $\epsilon_{s}=$ $-7.3 \mathrm{eV}$ for the $s$-orbital and $\epsilon_{p}=0$ for the triply-degenerate $p$-orbitals. The nearest neighbor pairs determined by the condition that the interatomic distance $R_{i j}$ being less than $1.75 \AA$ are assigned the hopping parameters $V_{s s \sigma}=-4.30 \mathrm{eV}, V_{s p \sigma}$ $=4.98 \mathrm{eV}, V_{p p \sigma}=6.38 \mathrm{eV}$ and $V_{p p \pi}=-2.66 \mathrm{eV}$. Further interactions are taken into account up to $R_{i j}=2.91 \AA$ which covers all the 2nd and 3rd nearest neighbors pairs by using scaled parameters $V_{s s \sigma}=-0.18 \alpha, V_{s p \sigma}=0, V_{p p \sigma}=0.35 \alpha$, and $V_{p p \pi}=-0.10 \alpha$ with the scaling parameter $\alpha=\left(3.335 / R_{i j}\right)^{2}$. This set of tight-binding parameters were successfully used earlier ${ }^{44,49}$ to calculate electronic properties of carbon nanotubes having deformed cross-sections. The original derivation of these empirical tight-binding parameters was performed by fitting to the ab-initio-calculated band structure of bulk graphite. ${ }^{50}$ The similar $s p^{2}$ coordination of atoms both in graphite and in SWNT makes this parametrization reason- 
ably valid for SWNTs as well. To test the tight-binding model we have calculated the ballistic conductance of infinite $(5,5)$ and $(8,0)$ SWNTs and found agreement with earlier calculations.

Using the above model we made conductance calculations for junction of parallel tubes connected with a carbon atoms, B-H2, force-free B-H2, B-H3, B-H4, H-H and B-H6 (i.e., the B-H5 junction which has a vacancy) configurations. Figure 10 shows the intertube equilibrium conductances of these systems. All conductance values for these structures do not exceed the unit quantum of conductance $G_{0}=2 e^{2} / \mathrm{h}$.

In Fig. 10(b), despite a large separation $(\sim 2.2 \AA)$ between two parallel tubes, significant intertube conductance, $0.5 G_{0}$, is achieved through the carbon atom that connects the tubes. The existence of the extra $\mathrm{C}$ atom between the tubes provides a ballistic channel for the conduction, however, the scattering mechanisms and the effect of the contact resistance limit its value below the unit quantum of conductance. The contact geometry of two parallel SWNTs that we studied can be contrasted to that of the telescopic arrangement of double wall carbon nanotubes (DWNT) as considered in Ref. 40. In the latter case, although the distance between the walls is comparable to our case, the intertube conductance they calculate is more than two orders of magnitude smaller due to the potential barrier between the walls of DWNT. In that case the system is in the tunnelling regime.

The contact force dependence of the intertube conductance in $(5,5)$ crossed nanotube junctions was investigated in calculations by Yoon et al., ${ }^{30}$ and a strong dependence on the contact force was calculated. On the contrary, in the present study using $(8,0)$ zigzag tubes, intertube conductance is inversely proportional with the contact force. For the B-H2 structure we calculated the equilibrium conductance as $0.28 G_{0}$. Conductance plots for B-H3 and B-H4 junctions support the previous conclusions reached through the analyses of contact structure and electronic potential. Hence the intertube conductance of highly compressed junctions is negligible due to a wide $\Phi_{B}$ intervening between tubes. The conductance of the junction which was relaxed after constraints on the B-H2 lifted and hence $F_{P}$ was released increases to $0.40 G_{0}$. A slight increase of $G$ can be attributed to the reduced deformation of contact in the absence of the constraints. The conductance of $\mathrm{H}-\mathrm{H}$ junction on the Fig. $10(\mathrm{~g})$ is comparable to those of $\mathrm{B}-\mathrm{H} 2$ and $\mathrm{B}-\mathrm{H} 2(\mathrm{r})$. The B-H5 junction, with a large $s$ and finite $\Phi_{B}$ between tubes, has $G \sim 0$. There is an empty gap of $\sim 0.5 \mathrm{eV}$ for ballistic conductance. However, upon the creation of vacancies, the potential barrier has collapsed and the calculated conductance increased to a value close to $0.8 G_{0}$. We note that the model used in calculating $G$ in Fig. 9 has short arms of $(8,0)$ SWNT leading to the junction. These short arms, which are also deformed under $F_{p}$ appear to be conductor, in spite of the fact that a long bare $(8,0)$ is a semiconductor.

\section{CONCLUSIONS}

In this work we present a first-principles study of junctions of parallel and crossing tubes using a periodically repeating supercell model. Junctions with different registry and radial deformation have been considered. Two tubes which are normally under a weak and attractive van der Waals interaction can be further linked by pressing them towards each other. Compressing tubes radially induces deformation and changes the circular cross section into an elliptical one. We showed that two parallel $(8,0)$ tubes can be linked by $\mathrm{C}-\mathrm{C}$ bonds between the tubes under weak as well as strong contact forces. The junctions of crossed nanotubes are studied by using a 2D grid model for a wide range of contact forces. It has been shown that certain physical properties do not vary continuously with the contact forces. The $\mathrm{C}-\mathrm{C}$ bonds interlinking the tubes can form under relatively weak contact forces. These bonds survive even after the contact forces are released. However, strong forces induce significant radial deformation and give rise to flattening of the tube surfaces. Once the surfaces becomes locally planar at the contact, the curvature effects diminish. At the end, interlinking bonds are broken and the spacing between chemically inactive flat surfaces increases as in graphite layers. Under these circumstances a finite potential barrier between tubes hinders ballistic electron transport from one tube to other. The potential barrier collapses if linking bonds between the tubes are present.

The formation of interlinking bonds can be enhanced by making the contact chemically active. This can be achieved by implementing imperfections, such as substitutional impurities with valencies different from four (such as B, N, P, etc.) or more conventionally by creating vacancies and divacancies. A single carbon atom between tube surfaces can also provide the interlinking. A single vacancy created on one of the tube surfaces makes the junction chemically active and establishes an $s p^{3}$-like bonding configuration. As a result, the potential barrier is collapsed through interlinking bonds. Our results related to junctions with vacancy explain how the stable crossbar structures can be fabricated by in situ processes. An alternative process to make crossbar, $\mathrm{T}$ and other types of junctions is to weld the tubes at the junction site by the chemisorption of atoms, which make stable chemisorption bonds. Here we mention the Ti atom, which is easily adsorbed and also can make thick coating of SWNTs. The calculation of quantum ballistic conductance through various junctions confirm the analysis based on potential energy and atomic structure for the behavior of the contact as a function of the contact force.

We also examined the 3D grid structure of tubes by using only limited supercell size consisting of $3 \times 3$ unit cells of $(8,0)$ zigzag tube laterally. Our results indicate that formation of linking bonds, stability and variation of electronic structure depend on the applied contact force. We believe that 2D and $3 \mathrm{D}$ grid structures can render a framework to integrate SWNT-based devices or functionalization by adsorption of molecules.

\section{ACKNOWLEDGMENTS}

This work was partially supported by the National Science Foundation under Grant No. INT01-15021 and TÜBİTAK under Grant No. TBAG-U/13(101T010). SC acknowledges partial support from Academy of Science of Turkey. 
${ }^{1}$ S. Iijima, Nature (London) 354, 56 (1991); S. Iijima, T. Ichihashi, and Y. Ando, ibid. 356, 776 (1992).

${ }^{2}$ N. Hamada, S. I. Sawada, and A. Oshiyama, Phys. Rev. Lett. 68, 1579 (1992).

${ }^{3}$ J. W. Mintmire, B. I. Dunlap, and C. T. White, Phys. Rev. Lett. 68, 631 (1992).

${ }^{4}$ X. Blase, L. X. Benedict, E. L. Shirley, and S. G. Louie, Phys. Rev. Lett. 72, 1878 (1994).

${ }^{5}$ M. S. Dresselhaus, G. Dresselhaus, and P. C. Eklund, Science of Fullerenes and Carbon Nanotubes (Academic, San Diego, 1996).

${ }^{6}$ R. Saito, G. Dresselhaus, and M. S. Dresselhaus, Physical Properties of Carbon Nanotubes (Imperial College Press, London, 1998).

${ }^{7}$ R. Saito, M. Fujita, G. Dresselhaus, and M. S. Dresselhaus, Appl. Phys. Lett. 60, 2204 (1992).

${ }^{8}$ J. W. G. Wildör, L. C. Venema, A. G. Rinzler, R. E. Smalley, and C. Dekker, Nature (London) 391, 59 (1998); T. W. Odom, J. Huang, P. Kim, and C. M. Lieber, ibid. 391, 62 (1998).

${ }^{9}$ O. Gulseren, T. Yildirim, S. Ciraci, and C. Kilic, Phys. Rev. B 65 , 155410 (2002).

${ }^{10}$ C. Dekker, Phys. Today 52, 22 (1999).

${ }^{11}$ For a comprehensive review on the electronic and transport properties of SWNTs see S. Ciraci, A. Buldum, and I. P. Batra, J. Phys.: Condens. Matter 13, 537 (2001); S. Ciraci, S. Dag, T. Yildirim, O. Gülseren, and R. T. Senger, ibid. 16, R901 (2004).

${ }^{12}$ A. Bezryadin, A. R. M. Verschueren, S. J. Tans, and C. Dekker, Phys. Rev. Lett. 80, 4036 (1998).

${ }^{13}$ C. J. Park, Y. H. Kim, and K. J. Chang, Phys. Rev. B 60, 10656 (1999).

${ }^{14}$ C. Kilic, S. Ciraci, O. Gülseren, and T. Yildirim, Phys. Rev. B 62 , R16345 (2000).

${ }^{15}$ O. Gülseren, T. Yildirim, and S. Ciraci, Phys. Rev. Lett. 87, 116802 (2001).

${ }^{16}$ E. Durgun, S. Dag, V. M. K. Bagci, O. Gülseren, T. Yildirim, and S. Ciraci, Phys. Rev. B 67, 201401 (2003); E. Durgun, S. Dag, S. Ciraci, and O. Gülseren, J. Phys. Chem. B 108, 575 (2004).

${ }^{17}$ J.-P. Selvetet, J.-M. Bonard, N. H. Thomson, A. J. Kulik, L. Ferro, W. Benoit, and L. Zuppitoli, Appl. Phys. A: Mater. Sci. Process. 69, 225 (1999).

${ }^{18}$ O. Gülseren, T. Yildirim, and S. Ciraci, Phys. Rev. B 65, 153405 (2002).

${ }^{19}$ S. J. Tans, A. R. M. Verscheuren, and C. Dekker, Nature (London) 393, 49 (1998); A. Bachtold, P. Hadley, T. Nakanishi, and C. Dekker, Science 294, 1317 (2001); T. Nakanishi, A. Bachtold, and C. Dekker, Phys. Rev. B 66, 073307 (2002).

${ }^{20}$ R. Martel, V. Derycke, C. Lavoie, J. Appenzeller, K. K. Chan, J. Tersoff, and Ph. Avouris, Phys. Rev. Lett. 87, 256805 (2001); $\mathrm{Ph}$. Avouris, R. Martel, V. Derycke, and J. Appenzeller, Physica B 323, 6 (2002).

${ }^{21}$ C. Zhou, J. Kong, and H. Dai, Appl. Phys. Lett. 76, 1597 (2000).

${ }^{22}$ H. Dai, E. W. Wang, Y. Z. Lu, S. Fan, and C. M. Lieber, Nature
(London) 375, 769 (1995).

${ }^{23}$ S. Dag, E. Durgun, and S. Ciraci, Phys. Rev. B 69, 121407(R) (2004).

${ }^{24}$ M. Menon and D. Srivastava, Phys. Rev. Lett. 79, 4453 (1997).

${ }^{25}$ G. Treboux, P. Lapstun, and K. Silverbrook, Chem. Phys. Lett. 306, 402 (1999).

${ }^{26}$ T. Yildirim, O. Gülseren, C. Kilic, and S. Ciraci, Phys. Rev. B 62, 12648 (2000).

${ }^{27}$ M. S. Fuhrer, J. Nygård, L. Shih, M. Forero, Y.-G. Yoon, M. S. C. Mazzoni, H. J. Choi, J. Ihm, S. G. Louie, A. Zettl, and P. L. Mceuen, Science 288, 494 (2000).

${ }^{28}$ S. Paulson, A. Helser, M. B. Nardelli, R. M. Taylor II, M. Falvo, R. Superfine, and S. Washburn, Science 288, 494 (2000).

${ }^{29}$ H. W. Ch. Postma, M. deJonge, Z. Yao, and C. Dekker, Phys. Rev. B 62, R10653 (2000).

${ }^{30}$ Young-Gui Yoon, Mario S. C. Mazzoni, H. J. Choi, J. Ihm, and S. G. Louie, Phys. Rev. Lett. 86, 688 (2001).

${ }^{31}$ M. Ouyang, J.-L. Huang, C. L. Cheung, and C. M. Lieber, Science 291, 97 (2001).

${ }^{32}$ A. Buldum and J. P. Lu, Appl. Surf. Sci. 219, 123 (2003).

${ }^{33}$ A. Pérez-Garrido and A. Urbina, Carbon 40, 1227 (2002).

${ }^{34}$ M. Terrones, F. Banhart, N. Grobert, J.-C. Charlier, H. Terrones, and P. M. Ajayan, Phys. Rev. Lett. 89, 075505 (2002).

${ }^{35}$ A. V. Krasheninnikov, K. Nordlund, J. Keinonen, and F. Banhart, Phys. Rev. B 66, 245403 (2002).

${ }^{36}$ J. W. Janssen, S. G. Lemay, L. P. Kouwenhoven, and C. Dekker, Phys. Rev. B 65, 115423 (2002).

${ }^{37}$ A. Nojeh, G. W. Lakatos, S. Peng, K. Cho, and R. F. W. Pease, Nano Lett. 3, 1187 (2003).

${ }^{38}$ A. Buldum and J. P. Lu, Appl. Surf. Sci. 215, 123 (2003).

${ }^{39}$ F. Cleri, P. Keblinski, I. Jang, and S. B. Sinnott, Phys. Rev. B 69, 121412(R) (2004).

${ }^{40}$ A. Hansson and S. Stafström, Phys. Rev. B 67, 075406 (2003).

${ }^{41}$ M. C. Payne, M. P. Teter, D. C. Allen, T. A. Arias, and J. D. Joannopoulos, Rev. Mod. Phys. 64, 1045 (1992). Numerical calculations have been performed using the VASP software: G. Kresse and J. Hafner, Phys. Rev. B 47, 558 (1993);G. Kresse and J. Furthmüller, ibid. 54, 11169 (1996).

${ }^{42}$ J. P. Perdew, J. A. Chevary, S. H. Vosko, K. A. Jackson, M. R. Pederson, D. J. Singh, and C. Fiolhais, Phys. Rev. B 46, 6671 (1992).

${ }^{43}$ H. J. Monkhorst and J. D. Pack, Phys. Rev. B 13, 5188 (1976).

${ }^{44}$ J.-C. Charlier, Ph. Lambin, and T. W. Ebbesen, Phys. Rev. B 54, R8377 (1996).

${ }^{45}$ R. Landauer, Philos. Mag. 21, 863 (1970).

${ }^{46}$ M. Buttiker, Phys. Rev. Lett. 57, 1761 (1986).

${ }^{47}$ S. Datta, Electronic Transport in Mesoscopic Systems (Cambridge University Press, Cambridge, 1995).

${ }^{48}$ A. P. Jauho, N. S. Wingreen, and Y. Meir, Phys. Rev. B 50, 5528 (1994).

${ }^{49}$ M. B. Nardelli and J. Bernholc, Phys. Rev. B 60, R16338 (1999).

${ }^{50}$ D. Tomanek and S. G. Louie, Phys. Rev. B 37, 8327 (1988). 